\title{
Tooth stabilization after dental trauma
}

\section{Alexandros Zeimpekis ${ }^{1}$, loanna Polichroniadou ${ }^{2}$, Dimitar Kosturkov ${ }^{3}$}

1. Military dentist at Naval and Veterans Hospital of Athens, BSc, DDS, MD candidate;

2. Graduate student in Faculty of Dental Medicine, Medical University Sofia

3. PhD, Assistant professor, Department of Conservative Dentistry, Faculty of Dental Medicine, Medical University Sofia

\begin{abstract}
Traumatic injuries of teeth can be seen very often and sometimes they can be challenging to treat. Different treatment approaches exist and each of them has specific indication. Dental trauma can be classified into three main categories: damage of hard dental tissues and pulp, lesions of hard dental tissues of pulp and alveolar tissues and lesions of periodontal tissues. In some of these cases, it is necessary to immobilize injured teeth. This is achieved by different means - rigid splints, semi-rigid splints and flexible immobilization splints. The first two groups are commonly used. Rigid splints are: wire splints, arch bar splints, acrylic cap splints, composite splints. Semi-rigid splints are: orthodontic wire and bracket splints, synthetic wire and composite splints, fiber splints, titanium trauma splint (TTS). The rigid splints are often used for a long time and for jawbone fractures to protect against normal tooth mobility. Instead, semi-rigid splints are a little more flexible and allow normal tooth mobility and are often used for a short period of time, such as in cases of avulsion, displacement, and dental fractures. The effects of splinting remain an open field of controversy, but it has been reported that splints used for a long time can cause
\end{abstract}


ankylosis and absorption. Even though, used in an adequate way, they are provide normal healing process and good treatment outcome.

Keywords: dental trauma, tooth, injury, fracture, splint, tooth mobility

\section{Introduction}

Dental trauma occupies humanity from the depths of the centuries as teeth were among the main organs not only for chewing, but also for arrest as well as for defense or attack. This makes us believe that people's teeth are going to be damaged through the past of the years. We have investigated from remains that teeth fractures of the primitive human were a frequent finding.

Additionally tooth splinting in the past years was made with rigid splints, for long periods of time up to a few months, mainly for jaw fractures [2]. In several cases of dental wound teeth stabilization with particular ways (splints) is one of the most important signs of the treatment of injured teeth. This is done to prevent incorrect placement and further injury of the pulp or periodontal junction during the healing phase $[1,2]$. The term splint has been defined by the American Endodontic Association as a rigid or flexible device or material used to support, protect or stabilize lost teeth, repopulate, fractured, or have been under various endodontic surgical procedures [3].

The purpose of the present study is to present the protocols associated with the immobilization of the teeth after a dental wound and the presentation of the different types of splints that can be used in the case of dental trauma.

This study was done using both a bibliographic review and the help of online research in scientific databases.

When a dental trauma of the permanent dentition will promote an immobilization for how long and what is the type of the used splint

Dental trauma can be classified into three main categories [4]

- Damage to hard dental tissues and pulp

- In lesions of hard dental tissues of pulp and alveolar tissue

- Lesions of periodontal tissues.

In some of these cases it is necessary to immobilize injured teeth. 


\section{With periodontal tissue involvment}

\section{- Subluxation}

Subluxation is an injury to the tooth supporting device resulting it's increased mobility, but without tooth movement of it's location. In this case, it is necessary to immobilize the injured tooth for up to two weeks with a flexible splint[5].

\section{- Lateral luxation}

It is defined as a displacement of the tooth in a direction other than axially, usually accompanied by fracture of the alveolar socket[3]. Immobilization is performed either with an acrylic splint or with a flexible wire splint to stabilize the injured tooth and to facilitate the patient for four weeks[4,6].

\section{- Intrusion}

The wedge is defined as the displacement of the tooth into the alveolar bone[3]. There are 3 pathways to re-position the injured tooth in its original position in the alveolar. This is done either by spontaneous repositioning or orthodontic repositioning or either with surgical repositioning. In the case of surgical sunrise, a flexible splint is placed for four weeks[4].

\section{- Extrusion}

Defined as the partial displacement of the tooth outside its original position[3]. It is required to immobilize tooth for two weeks using either a flexible acrylic splint or a flexible wire splint[4].

\section{- Avulsion}

The case of avulsion is a separate class of dental trauma, and it cannot be missed by our reference. As avulsion is defined the complete displacement of the tooth outside the alveolar bone[3].

The basic separation in treatment of the tooth and immobilization of the tooth (which is part of the treatment) is whether the apex of the tooth is open or closed. Perhaps the most important factor that determines in part the expected result is the extraoral dry time[4].

More specifically, in the case of avulsion of tooth with a closed apex and extra oral time in physiologic storage media or osmolality balanced media (milk,saline,saliva or hank's balanced salt solution) less than 60 minutes the suggested immobilisation is a flexible splint for 2 weeks. For incidents that arrived at the clinic after 1 hour passes from the incident, the duration of use of the splint is extended to 4 weeks[6].

As for cases with open apex if they come to the clinic before 60 minutes and the tooth has been kept in a humid environment, the immobilization proposed is the use of a flexible splint for 2 weeks, instead of coming after 60 minutes that the duration should be extended to 4 weeks[4]. 


\section{Without periodontal tissue involvement}

- Crown-root fracture without pulp involvement and crown- root fracture with pulp involvement

The crown-root fracture without pulp involvement is a fracture of enamel, dentin, and cysteine without the junction of the pulp, but without losing the structure of the tooth. The pulp-entrapped crown-root fracture is a fracture of enamel, dentin, cysteine with loss of the structure of the tooth[3,4].

In both cases a temporary immobilization of the injured tooth in the emergency can be used until we have a definitive treatment plan[4].

\section{- Root fractures}

It is necessary to immobilize the affected tooth with a flexible acrylic splint or a flexible wire splint for 4 weeks if the fracture is in the middle or in the upper third of the root. If the fracture is in the cervical tremor of the root then the immobilization can last up to 4 months[4].

\section{Damage of hard dosage tissues and facial castle}

\section{- Dentoalveolar fracture}

A dental alveolar fracture may or may not include the alveolar socket. Immobilization involves the stabilization of the fractured section with a flexible splint for four weeks[4].

\section{- Jaw fractures}

It is a fracture involving the upper or lower jaw and often the alveolar bone. It may or may not include the (alveolar socket). Immobilisation of this fracture is done with (intermaxillary) splint for four weeks [4, 5, 7].

\section{Desirable characteristics of a dental splint}

The reaquirements of a splint should be [7-20] :

1. Semi-rigid and passive in normal tooth movement,

2. Allow pulp sensibility testing and access for endodontic treatment

3. Avoid interference with closing,

4. Not injuring the lips and periodontal tissues,

5. Fulfill aesthetic appearance

6. Contributing to and facilitating the maintenance of oral hygiene

7. Comfortable and easily applied and removed

8. Not to interfere with speech and occlusion. 


\section{Splint types}

Many different types and ways of splinting exist and are used in clinical practice. A separation would be rigid splints, semi-rigid splints, and flexible immobilization splints [21,22]

\section{Rigid splints}

- Wire splints

- Arch bar splints

- Acrylic cap splints

- Composite splints

\section{Semi-rigid splints}

- Orthodontic wire and bracket splints

- Synthetic wire and composite splints

- Fibre splints

- $\quad$ titanium trauma splint (TTS)

\section{Wire splints}

These splints are used when there are many teeth missing or during mixed dentition. A soft wire is used around the teeth for 8 consecutive cycles. This type of immobilization is used when the immobilisation is indicated for a short time. Its drawbacks are that iron wire breaks easily and also it is difficult to achieve oral hygiene with the result of gingivitis. Having in mind all that ,it is recommended to use them only for a few days[22,24].

\section{Arch bar splints}

Their first use was made by Hammond in the 1870s. The splints for maxillary and mandibular fractures consist of a metal arched beam in the shape of the dental arc which is secured in place by means of connecting wires. Its main disadvantage is that it is rigid and therefore its use in the case of dental trauma is limited[24].

\section{Acrylic cap splints}

This splint is made of acrylic and has been used to treat displaced teeth in conjunction with alveolar fractures. Making this splint straight in the patient's mouth can be very inconvenient for him and that's why we prefer to make it extra oral in a model. But even this has a negative as we must avoid getting teeth impression in case of dental trauma[24]. 


\section{Composite splints}

These splints are made of a strip of synthetic material that is placed directly on the prosthetic surfaces of the teeth to be cast. The resin is placed continuously on the lip surfaces of the crown of all the teeth to be splinted. Such splints are easy to construct, but tend to break easily at the points joining the teeth between them when they receive clumping load and therefore they can't be used for long term immobilization[24]. Furthermore they cause more gingival irritation compared with wire and composite, orthodontic bracket and titanium trauma splint[20].

\section{Orthodontic wire and bracket splints}

These splints consist of braces placed in the middle third of the surface of the teeth with photopolymerizable composite resin. A soft $0,3 \mathrm{~mm}$ cross-section wire passes through a bracket to a bracket to connect all the teeth. These splints, compared to the titanium trauma splints, cause greater gum irritation[20] The clinician should also be careful not to transfer orthodontic forces to the teeth since the orthodontic wires are never passive and generate forces up to $27.33 * 0.01 \mathrm{~N}$ which are capable to shift the incisors[23]. The advantage of the orthodontic wire splint is the immediate movement and replacement of teeth when needed.

\section{Wire and composite splints}

This method was applied by O'Riordan and his associates who used a fine flexible wire of 0.3-0.4 mm diameter as a splint. This semi-rigid splint is stabilized with a photopolymerizable composite resin[26]. It is the most used splint to immobilize injured teeth as it meets all the requirements of modern clinical dentistry. It can also be used in almost all types of dental wounds.

The resin is placed above the wire on the individual side of the tooth, but does not extend into the interdental areas[24]. The amount of resin deposited on the wire will affect the rigidity of the splint wire as it reduces the free and unformed portion of the wire. Another factor calculated for the rigidness of the splint is the diameter of the wire. The smaller the diameter of the wire used the more flexible the splint will be[21]. It is noted that a 0.3-0.4 mm diameter wire was found to be more flexible than a $0.5 \mathrm{~mm}$ diameter wire[23].

Cases where this type of casting cannot be used are in crowns, porcelain veneers, and in large fillings as there is no possibility of rooting the surface of these restorations as well as when there is a lack of other neighbor teeth. It is difficult, but not impossible, to place this kind of splinting on tooth with small crown[24].

One issue that raises concerns is the bond formed between the wire and the resin. It has been found that if sandblasting the wire improves the bond between resin and stainless steel wire.

\section{Fiber splints}

These splints are resin based and additionally contain fibers designed to enhance their properties. Main advantage of the fiber splints is that they do not need a dental laboratory to make them, but they can be connected directly to the teeth. They have good durability and are repairable. 
Their usefulness is quite heterogeneous and depends on the nature of the fibers used, the geometrical characteristics of the fibers constituting the fibers (one-way, mesh, wooven arrangement) and the resinous material covering them.

The most used fiber splints are glass fiber reinforced filaments, high strength polyethylene fiberglass splints and Kevlar fiber splints[22,27].

\section{Titanium trauma splints (TTS)}

TTS is a new device developed by von Arx et al. It is made of solid titanium with a thickness of $0.2 \mathrm{~mm}$ and a width of $2.8 \mathrm{~mm}$ (Medartis AG, Basel, Switzerland). It has a diamond shape which makes it more flexible in order to allow normal tooth movement. It can be easily adjusted to the curved contour of the dental arc with no additional means for its application. It is also easy to cut with any cutting instrument. The amount of resin used to attach the splint to the tooth is small while the rhomboid shape makes it easier to remove the splint after use. In order to splint from canine to canine, a TTS splint of about $52 \mathrm{~mm}$ is required. Because it is thin and flexible it can be easily adjusted to the desired position.

A thin layer of a thin-layer resin is placed in the rhombic opening of the splint after use of the etching and the adhesive agent[28].

In a comparative study by Von Arx et al ,TTS is the easiest and takes less time to place ( $8.5+/$ - $0.76 \mathrm{~min})$ compared to wire and composite splint $(10.1+/-1.29 \mathrm{~min})$ or the bracket splint $(13.1+/-0.94 \mathrm{~min})$.. Finally, TTS is more comfortable for patients because it is less bulky and does not affect speech or oral hygiene[29].

\section{Comparative study of four patients using the most used teeth splints as of the comfort of the patient}

\section{The comparison was made between the following splints}

- Wire composite splint WCS

- Button-bracket splint (BS)

- $\quad$ Resin-splint (RS)

- Titanium trauma splint (TTS)

The parameters for the comparison of these four types of splint are:

- The sensitivity of the toothed teeth

- Gingival irritation

- The irritation of the lips

- The inability to articulate speech, inability to eat and failure to perform oral hygiene or difficulty

The study showed that all four splint types allow normal tooth mobility, but TTS and WCS allowed a mobility closer to that of the teeth, and RS significantly reduced tooth mobility. 
Regarding their placement time from the dentist, it was significantly smaller for the TTS.

It was observed that there was no statistical difference between the four types of splint in the parameters of inability to eat and irritation of the gums at least in a short time after their placement.

However, in the long run, RS showed an increased gum disease compared to other splints. The sensitivity of the teeth and lips was greater on the first day and continued to decrease as the days progressed.

Statistically significant differences in the sensitivity of the teeth were found between BS (increased) and WCS only for the first day after their application. Also a difference was found in the sensitivity of the lips between BS and WCS and RS.

Regarding the inability to articulate speech, significant differences were found on the first day between BS compared to all other splints.

Finally, oral hygiene is significantly reduced in BS compared to all other splints irrespective of their period of use[20].

\section{Comparative study of rigid with the most further used teeth splints}

Comparing the most used stabilization splints after dental trauma in terms of their rigidity are classified from flexible to rigid.

An acrylic resin model was used in this study and the study was conducted in vitro. Teeth that resembled injury were the central incisors and had horizontal and vertical mobility. The lateral incisors as well as the canines resembled the unfractured teeth. Four composite splints, three composite splints, 1 titanium traumatic splint, 1 titanium ring splint, 1 bracket splint, and 2 Schuchardt splints. In all injured teeth and for all splints there was a remarkable effect of the splinting on the horizontal and vertical dimensions.

According to the existing protocols and within the limits of the in vitro study, we can note that rigid and semirigid splints such as the titanium trauma splint and wire-composite splints are suitable for immobilizing teeth that have undergone dislocation as well as root fractures. On the contrary, rigid splints such as the titanium ring splint can be used for the treatment of dentoalveolar fractures[21].

\section{In Conclusion}

It seems that rigid splints are often used for a long time and for jawbone fractures to protect against normal tooth mobility.

Instead, semi-rigid splints are a little more flexible and allow normal tooth mobility and are often used for a short period of time, such as in cases of avultion, displacement, and dental fractures.

Rigid splints used between 2-6 weeks are believed to increase the severity and frequency of external absorption, while semi-rigid splints offer better protection against root absorption. 
Finally, the effects of splinting remain an open field of controversy, but it has been reported that splints used for a long time can cause ankylosis and absorption[7-9].

\section{References}

1. Andreasen JO, Andreasen FM. Textbook and color atlas of traumatic injuries to the teeth, 3rd edn. Mosby: Munksgaard; 1994.

2. Kehoe JC. Splinting and replantation after traumatic avulsion J Am Dent Assoc 1986;112:22430.

3. American Association of Endodontists .An annotated glossary of terms used in endodontics 7th edn .Chicago : American Association of Endodontics 2003,15

4. www.dentaltraumaguide.org

5. Andreasen JO, Andreasen FM, Andersson L, eds. Textbook and Color Atlas of Traumatic Injuries to the Teeth. 4th edn. Copenhagen, 2007:404-410.

6. International Association of Dental Traumatology (IADT) dental trauma guidelines Revised 2012

7. Andreasen JO. Traumatic injuries of the teeth. Copenhagen: Munksgaard, 1972:154.

8. Endodontics colleagues for excellence:The treatment of the traumatic dental injuries (summer 2014)

9. Mandel U, Viidik A. Effect of splinting on the mechanical and histological properties of the healing periodontal ligament after experimental extrusive luxation in the monkey. Arch Oral Biol 1989;34:209-17

10. Ram D, Cohenca N. Therapeutic protocols for avulsed permanent teeth: review and clinical update. Pediatr Dent 2004;26:251-5.

11. Andreasen JO. The effect of splinting upon periodontal healing after replantation of permanent incisors in monkeys. Acta Odont Scand 1975;33:313-23.

12. Kristerson L, Andreasen JO. The effect of splinting upon periodontal and pulpal healing after autotransplantation of mature and immature permanent incisors in monkeys. Int J Oral Surg 1983;12:239-49.

13. Barbakow FH, Cleaton-Jones PE. Experimental replantation of root canal-filled and untreated teeth in the Vervet monkey. J Endodon 1977;3:89- 93.

14. Kehoe JC. Splinting and replantation after traumatic avulsion. J Am Dent Assoc 1986;112:22430

15. Yasuda T, Kinoshita M, Abe M, Shibayama Y. Infavorable effect of knee mobilization on Achilles tendon healing in rabbits. Acta Orthop Scand 2000;71:69-73.

16. Massler M. Tooth replantation. Dent Clin 1974;18:445-52.

17. Hinckfuss SE, Messer LB. Splinting duration and periodontal outcomes for replanted avulsed teeth: a systematic review. Dent Traumatol 2009; 25: 150-157

18. Flores MT, Andreasen JO, Bakland LK. Guidelines for the evaluation and management of traumatic dental injuries. Dent Traumatol 2001;17:193-6.

19. Gregg TA, Boyd DH. UK National Clinical Guidelines in Paediatric Dentistry. Treatment of avulsed permanent teeth in children. Int J Paediatr Dent 1998;8:75-81.

20. Filippi A, von arx T, Lussi A. Comfort and discomfort of dental trauma splints-a comparison of a new device (TTS) with three commonly used splinting techniques. Dent Traumatol 2002;18:275-280.

21. Christine Berthold, Alexandra Thaler, Anselm Petschelt .Rigidity of commonly used dental trauma splints .Dental Traumatology 2009; 25: 248-255 
22. B Kahler, ${ }^{*} \mathrm{~J}-\mathrm{Y} \mathrm{Hu}, \dagger \mathrm{CS}$ Marriot-Smith, $\neq$ GS Heithersay§ .Splinting of teeth following trauma: a review and a new splinting recommendation.Australian Dental Journal 2016; 61:(1 Suppl) 59_ 73

23. Prevost J, Louis P, Vadot J, Granjon Y. A study of forces origi- nating from orthodontic appliances for splinting of teeth. Endod Dent Traumatol 1994;10:179-184.

24. Oikarinen K. Tooth splinting: a review of the literature and consideration of the versatility of a wire-composite splint. Endod Dent Traumatol 1990;6:237-2

25. Oikarinen K. Comparison of the flexibility of various splinting methods for tooth fixation. Int $\mathrm{J}$ Oral Maxillofac Surg 1988;17:249-252

26. O'RIORDAN MW , RAI.STROM CS, DOERR SE. Treatment of avulsed permanent teeth: an update. JADA 1982; 105: 10283

27. Andreasen JO, Andreasen FM, Mejare I, Cveck M. Healing of 400 intra-alveolar root fractures. 2. Effect of treatment factors such as treatment delay, repositioning, splinting type and period and antibiotics. Dent Traumatol 2004;20:203-211.

28. Thomas von Arx, Andreas Filippi,Daniel Buser.Splinting of traumatized teeth with a new device: TTS (Titanium Trauma Splint).Dent Traumatol 2001; 17: 180-184

29. Thomas von Arx, Andreas Filippi, Adrian Lussi.Comparison of a new dental trauma splint device (TTS) with three commonly used splinting techniques.Dent Traumatol 2001; 17: 266-274

\section{Corresponding author:}

Ioanna Polichroniadou, Faculty of Dental Medicine, Medical University Sofia, email: iwannapolichroniadou@gmail.com 